\title{
Estrategia Metodológica para la Visualización Digital de Patrones Aerodinámicos Presentes en la Morfología Urbana y su Incidencia en el Uso Estancial de los Espacios Públicos
}

\author{
Methodological Strategy for the Visualization of Aerodynamic Patterns in the Urban Morphology and \\ their Impact on the Use of Public Spaces
}

\author{
Carlos Bustamante Oleart \\ Universidad Finis Terrae Chile \\ cbustamante.o@gmail.com \\ Paulo K. Ogino Altamirano \\ Universidad Finis Terrae, Chile \\ paulo.ogino@gmail.com
}

Ester Higueras García

Universidad Politécnica de Madrid, España

ester.higueras@upm.es

\begin{abstract}
In relation to cities, wind is one of the less studied meteorological parameter. Constant vertical rate variations from meso scale to micro scale, altogether with strenght, direction, velocity of the urban canyon, makes the wind a complex subject of study. To achieve this, a methodological strategy that addresses the wind's multi dimensionality was raised. It was aplied to Punta Arenas, the southernmost city in the world, where most of the time winds come $90 \%$ from the same direction with speeds reaching $128 \mathrm{~km} / \mathrm{h}$. Wind's constant directional behavior allows the recognition of areodynamic phenomena produced, in the first instance, for the wind profiles influenced by the urban rugosity and then, at morphologycal level, the aerodynamic behavior of the layered fluids over the building bodies, generating a regular pattern between solids and fluids. The strong winds in cities with cold climate influence the use of public spaces, which, not being designed under this conditions, do not develop proper levels of thermal comfort.
\end{abstract}

Keywords: Aerodynamics; Urban morphology; Public space; Information visualization.

\section{Contexto de Investigación}

La presente ponencia se inscribe dentro de una investigación mayor de la Tesis Doctoral de Carlos Bustamante, que desarrolla una propuesta metodológica para la verificación y modelamiento de los principios de la aerodinámica aplicada a las formas urbanas y que buscarían mejorar tanto la eficiencia energética de los cuerpos edificados entorno a los espacios públicos como también aumentar el uso estancial de estos y su permanencia en ellos.

Son numerosos los acuerdos, directrices y legislaciones que se han aprobado para lograr un desarrollo más sostenible de los crecimientos urbanos. El urbanismo bioclimático establece las claves para conseguir que las ordenaciones estén integradas en su entorno, se gestionan eficazmente los recursos que facilitan una mejor calidad de vida a sus usuarios. (Higueras, 2004)

Dos de los factores más relevantes en el urbanismo bioclimático son el "sol y el viento", siendo las temáticas solares las más estudiadas en todas sus escalas, dado que el viento por su complejidad de comportamiento lo vuelve más difícil de medir y estudiar. Aumentando los costos de investigación.

Los estudios científicos sobre el conocimiento del viento se inician en la última mitad del siglo XX. Estos abarcan una gran diversidad de disciplinas. Siendo las tres más relevantes la vinculadas a la arquitectura (urbanismo), Geografía (meteorología) e ingeniería (aerodinámica). Estas tres disciplinas, a su vez han genero una variedad de métodos de estudio ya sea a través de sistemas de medición en situ, túneles de viento, sistemas de fluidos computacionales, cálculos numéricos y estadísticos del comportamiento del viento. Provocando también una variedad de metodologías de análisis; mapificaciones, gráficas, diagramas, esquema espectrales del comportamiento del viento, etc. Siendo la gran mayoría de estas metodologías estrategias de visualización de la información, buscando representar un fenómeno (el viento) que solo se manifiesta a través de otros elementos (nubes, remolinos, movimiento de los árboles, banderas, etc.)

En la última década y gracias a las nuevas tecnologías digitales se ha avanzado mucho en el comportamiento del viento en la micro escala de la ciudad. Esto ha provocado una serie de investigaciones multidisciplinares que buscan desarrollar indicadores y guías de diseño urbano que permitan corregir y producir proyectos más sostenibles. 
Estos estudios apuntan a mejorar los sistemas de ventilación urbana a través de un manejo de la morfología urbana incorporando aspectos en sus diseños que contribuyan a modelar el flujo del viento por la ciudad. Dicha metodología no solo construye una caracterización del comportamiento del viento a nivel urbano sino también un campo de medición que permite parametrizar los incides apropiados de circulación del aire en los tejidos urbanos.

La pregunta que comanda este trabajo es saber ¿Cómo estas metodologías multidisciplinares para estudiar el viento, desarrolladas a través de estrategias de visualización de la información, podrían orientar el diseño urbano de manera apropiada en ciudades con climas fríos y vientos fuertes?

Sobre la base de estas premisas, el proyecto de investigación busca innovar en un modelo metodológico propio sobre el estudio del viento, buscando a través de un método multi sistémico de visualización y cruce de información, la verificación de la incidencia del viento en el espacio público.

\section{Caso de Estudio}

Se propone estudiar cómo caso de estudio a la ciudad de Punta Arenas, Chile. Es la ciudad más austral del mundo. La rosa del viento indica que durante todo el año se tiene un viento predominante del oeste seguido de pequeñas variaciones del noroeste y suroeste. Y por otro lado la trama urbana está en la misma dirección que el viento predominante.

La ciudad de Punta Arenas tiene un centro depresionario que atrae grandes masas de aire con vientos de cierta violencia; la media alcanza los $14 \mathrm{Km} / \mathrm{h}$ con variaciones entre los 9 y $22 \mathrm{Km}$. Con máximas absolutas de hasta $120 \mathrm{Km} / \mathrm{h}$ (diciembre 1998). Su clima es estepario frío con temperaturas bajas, con promedios térmicos anuales del orden de los $6^{\circ} \mathrm{C}$. En el invierno las precipitaciones caen en forma de nieve (Urbe, 2000).

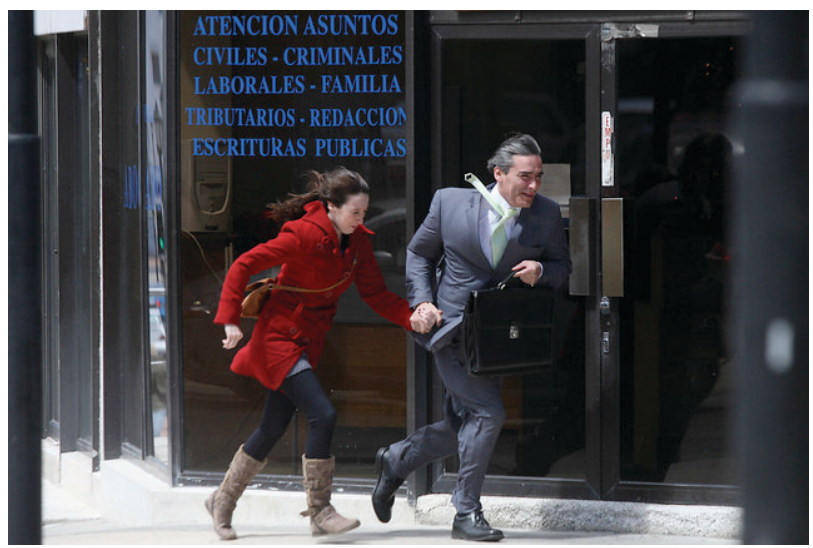

Figura 1: Ráfagas de viento que superan los $120 \mathrm{Km}$ por hora teniendo que colocar cuerdas en la ciudad (Fuente Archivo Agencia Uno - 2011)

\section{Metodología y Trabajo de Campo}

La primera instancia fue desarrollar un levantamiento digital y elaborar un modelo 3D del casco fundacional de la ciudad con el fin de usar dicho modelo como base para el estudio termodinámico.

La segunda instancia de estudio fue la definición y acotamiento del área de estudio. Se desarrolló un "Taller de Rehabilitación Urbana Eficiente" en la Escuela de Arquitectura de la Universidad de Magallanes desde la metodología de la agenda 21. El taller fue desarrollado por un equipo de académicos pertenecientes al "Magister de medio ambiente y Arquitectura bioclimática" (MAYAB) desarrollado en la Universidad Politécnica de Madrid. El taller permitió reconocer 5 áreas potenciales de trabajo, donde existía tanto la conciencia colectiva y las evidencias necesarias del encuentro entre el viento y los espacios de encuentro públicos.

La tercera instancia de estudio fue analizar la incidencia solar y térmica tanto a nivel planta de la ciudad como en sus fachadas, para ello se realizaron dos etapas; Primero el estudio en planta de la ciudad reconociendo los conos de sombra y áreas de mayor asolamiento según volumetría de la ciudad para luego pasar a la segunda etapa de verificación y reconocimiento del comportamiento térmico de las fachadas a través de una Cámara térmica profesional PCE-TC 3.

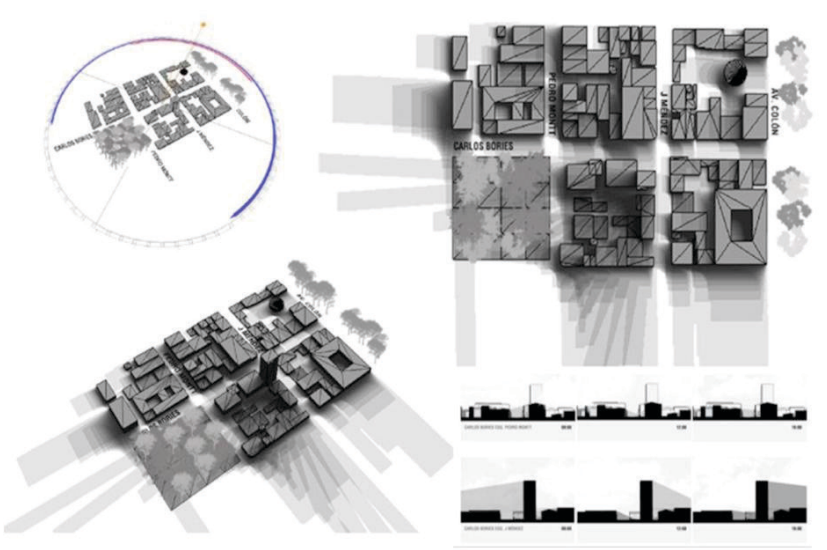

Figura 2: Análisis solar desarrollado por partes de la ciudad comprendiendo según solsticios las áreas más asoleadas y más sombrías. (Fuente propia)

- La cuarta instancia fue estudiar la incidencia eólica dentro de la morfología urbana tanto a nivel planta como en su cañón urbano. Al igual que las instancias anteriores se desarrollaron dos etapas; la primera fue a través de tres estaciones meteorológicas móviles Datalogger del tipo NOMAD de Secondwind, lo que permitió in situ medir el comportamiento del viento dentro de la ciudad en su fuerza y dirección. Para luego compararlo con la segunda etapa que fue desarrollar a través de un simulador de dinámicas de fluido CFD, (Autodesk Vasari) la dinámica del viento y posteriormente se realizó una simulación más detallada mediante Ecotect. 


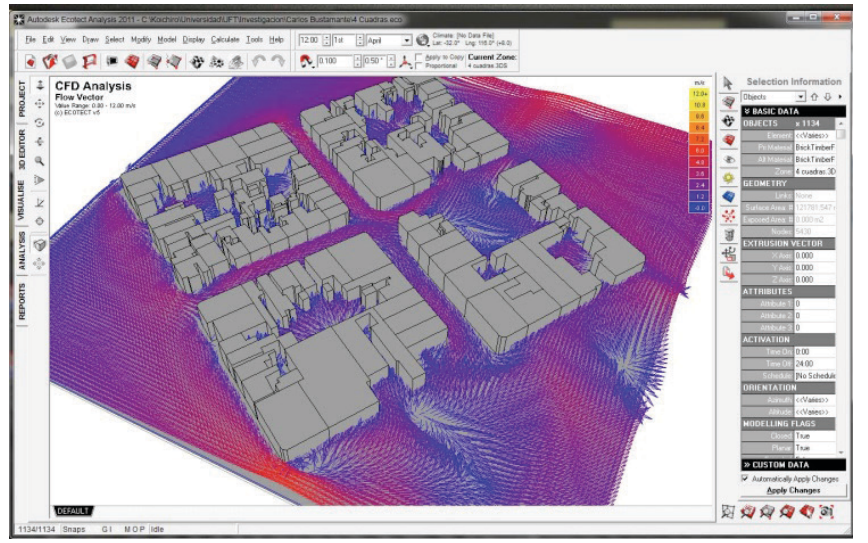

Figura 3: Cuarta instancia, estudio de la incidencia eólica dentro de la morfología urbna, etapa 2; a través de un CFD, simulador de dinámicas de fluido (Autodesk Vasari)

(Fuente: Elaboración propia).

- La quinta instancia fue desarrollar un estudio sobre el uso estancial de los espacios públicos reconociendo con ello, las áreas de mayor y menor uso. Para ello se optó por desarrollar el levantamiento de información en los meses de marzo, abril y mayo del 2012. Dado que dichas fechas obedecen al retorno habitual después de las vacaciones a la ciudad concediendo también con el inicio del año escolar y por ente a una normal uso del espacio público. Por otro lado la estación de otoño pese a no tener mucha presencia (solo existen dos estaciones muy marcadas verano e invierno) durante otoño se tiene la más alta frecuencia de viento desde la misma dirección, lo que permite mantener una constante importante.

- La sexta instancia de estudio corresponde a un trabajo de encuestas elaborado a través de 12 preguntas que busca comprender desde la memoria colectiva y experiencia vivencial la relación que tiene el habitante con el viento. Comprendiendo aquí una realidad aclimatada y acostumbrada a dicho fenómeno y que han desarrollado costumbres en torno a ello.

Finalmente todos esto datos fueron cruzados con un estudio paralelo sobre el uso estancial de los espacios públicos que permitió reconocer las áreas de mayor y menos uso que al superponer la información generada por los otros estudios permitió visualizar y verificar el nivel de incidencia del viento en los usos estanciales de los espacio público en el casco fundacional de la ciudad de Punta Arenas.

Respecto del uso del software mencionado anteriormente, se hicieron pruebas generales con Autodesk Vasari y posteriormente se realizó una simulación más detallada mediante Ecotect. Se alimentó al software con la información climática de Punta Arenas y posteriormente se procedió a realizar la simulación. Esta se realizó por bloques y también en forma general, donde los bloques constituían zonas de $2 \times 2$ cuadras y el modelo general era la suma de estos bloques.

\section{Aplicación del CFD.}

El CFD es una excelente herramienta para generar de manera simulada conocimiento del comportamiento tanto existente o posible dentro de una estructura urbana, lo que permite "experimentar" cómo este comportamiento influye en el habitar del espacio urbano

Durante el desarrollo del estudio se utilizaron los modelos 3D creados para realizar varias pruebas en diferentes escalas, desde manejo de bloques de cuadras hasta una escala mayor, en base a las zonas de estudio definidas. De esta manera se obtuvieron simulaciones de los flujos de aire que se producirían en las zonas seleccionadas y se pondrían en contraste respecto del actual uso de los espacios públicos
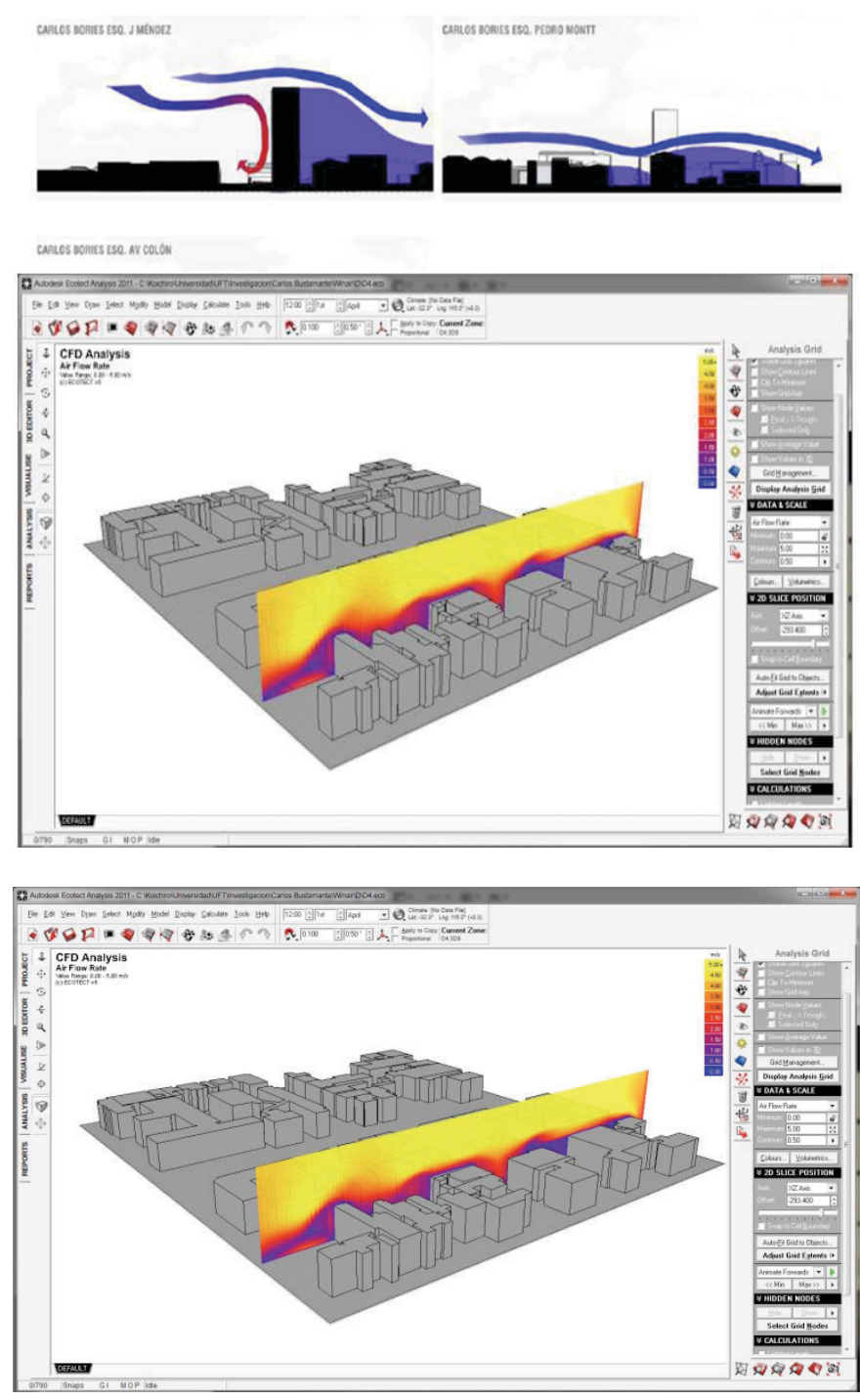

Figura 4: Análisis en corte por bloque urbano para verificar el comportamiento del viento a través de un CFD, simulador de dinámicas de fluido (Autodesk Vasari) (Fuente: Elaboración propia). 


\section{El Futuro del Diseño Urbano Basado en Simulaciones}

La modelización de los patrones de flujo dentro de las ciudades es una configuración compleja y no pueden aun dar lugar a normas explícitas. Para centrarse en el impacto geométrico del flujo eólico al interior de la ciudad, se sugiere hacer hincapié en tres instancias de complejidad.

- Evaluar con más precisión la encrucijada de calles reconociendo los comportamientos de remolinos laterales en el cañón urbano y como estos afectan a la comodidad peatonal.

- Visualizar mejor las incidencias de cambio de alturas dentro de la trama urbana, saliéndose de la rugosidad de la ciudad y su impacto dentro del espacio público.

- Reconocer las diferencias de comportamiento térmico entre veredas de las calles que están transversalmente al viento predominante

Si bien hace ya un buen tiempo se vienen realizando simulaciones para estudiar el comportamiento de los diseños, éstas se han desarrollado principalmente en el ámbito de la micro escala, el diseño de objetos, productos y edificaciones. Los CFD y otras aplicaciones de simulación permiten no solo graficar los flujos simulados sino también estudiar alternativas de diseño al momento de proponer intervenciones urbanas. La macro escala ya está siendo estudiada desde la simulación y el conocimiento obtenido mediante la graficación de estas simulaciones permitirá el mejor planteamiento de las ubicaciones y formas más adecuadas para los espacios públicos, para de esta manera desarrollar diseños más amables con el habitante.

"El viento ha estado durante siglos postergado pero ya vendrá su esplendor, así como lo tuvo el sol en la arquitectura moderno."

(Casanueva, 2004)

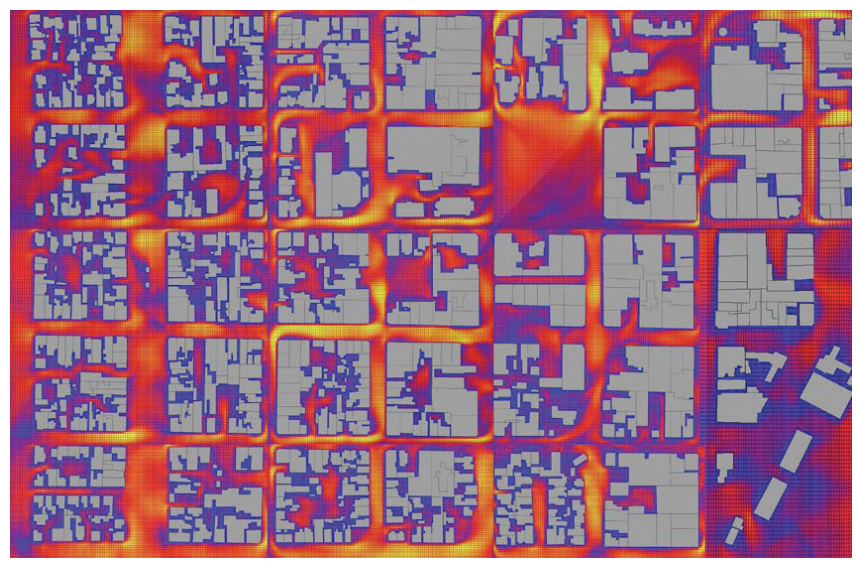

Figura 5: Imagen de todo el casco histórico de la ciudad de Punta Arenas a través de un CFD, simulador de dinámicas de fluido (Autodesk Vasari)

\section{Agradecimientos}

EL proyecto de investigación desarrollado a la luz de la tesis doctoral del arquitecto Carlos Bustamante aún se encuentra en proceso de cruce de información y conclusiones finales.

El trabajo de investigación que se ha realizado durante tres años ha reunido a cuatro instituciones universitarias ; La Universidad Finis Terrae a través de los fondos iniciales de investigación de Vicerrectoría Académica generando la base teórica de esta, participado la directora de la Escuela de Arquitectura Magdalena Sierra y los académicos Margarita Jans y Paulo Ogino, La Universidad de Magallanes a través de su director de Escuela de Arquitectura Fabián Barros y del director del Centro de Estudios de los Recursos Energéticos (CERE) Humberto Vidal facilitando todos los equipos y estaciones meteorológicas portátiles para la medición en terreno del viento. La Universidad Politécnica de Madrid a través de la directora de la tesis doctoral Ester Higueras y la implementación de un "Taller de Rehabilitación Urbana Eficiente" programa dependiente del MAYAB desarrollado de manera inédita en la ciudad de Punta Arenas durante el 2012 junto a las académicas Emilia Román y Teresa Eiroa. Y finalmente la Universidad San Sebastián, quien a través de su curso "formas aerodinámico" ha permitido precisar y abrir nuevo campos de investigación.

\section{Referencias}

Casanueva, M., C. (2004). Entrevista personal a la luz del libro:

De los campos de abstracción y los elementos para una arquitectura experimental. Editores; Mariana Vergara y Carlos Bustamante, Universidad Finis Terrae.

Higueras G., E.,(2006). Urbanismo Bioclimático. GG.

Urbe. (1999). Levantamiento Plan Seccional Avenida Costanera del Estrecho. Memoria. Ilustre municipalidad de Punta Arenas. Chile. 